\title{
Stretch and orientational mode decoupling in relaxation of highly stretched polymer melts
}

\author{
Anine L. Borger $\odot$ \\ Niels Bohr Institute, University of Copenhagen, 2100 Copenhagen, Denmark \\ Qian Huang $\odot$ and Ole Hassager $\odot$ \\ Department of Chemical and Biochemical Engineering, Technical University of Denmark, 2800 Kongens Lyngby, Denmark \\ Jacob J. K. Kirkensgaard $\odot$ \\ Niels Bohr Institute, University of Copenhagen, 2100 Copenhagen, Denmark \\ and Department of Food Science, University of Copenhagen, 1871 Frederiksberg, Denmark \\ Kristoffer Almdalø \\ Department of Chemistry, Technical University of Denmark, 2800 Kongens Lyngby, Denmark \\ Kell Mortensen $\odot$ \\ Niels Bohr Institute, University of Copenhagen, Denmark 2100 Copenhagen, Denmark
}

(Received 13 March 2020; accepted 3 August 2020; published 22 October 2020)

\begin{abstract}
We study the relaxation of linear polymer molecules following fast uniaxial extension. Polystyrene melts of $M_{w}=80 \mathrm{~kg} / \mathrm{mol}$ are elongated at a Rouse-Weissenberg number $\mathrm{Wi}_{R}=1.5$ to Hencky strain $\varepsilon=3$, where steady state is approached using a filament stretch rheometer. Samples were quenched for different duration of stress relaxation at constant Hencky strain to preserve molecular conformation by rapid cooling below the glass transition temperature. Expansion of ex situ small-angle neutron-scattering data in spherical harmonics shows experimental evidence for the decoupling of local and global relaxation phenomena from highly stretched states. This work demonstrates both that the decoupling can be seen in the spherical harmonics expansion and that it can be seen even for short chains provided the initial state is highly oriented.
\end{abstract}

DOI: 10.1103/PhysRevResearch.2.043119

\section{INTRODUCTION}

During industrial processing, polymer melts are often exposed to rapid, large deformations that result in molecular conformations far from equilibrium [1]. Understanding the relaxation dynamics of polymer chains from such highly oriented states is relevant from both a polymer processing and a fundamental polymer physics point of view.

One approach to studying the nonequilibrium dynamics is to expand from models of equilibrium dynamics. The equilibrium dynamics of polymer melts is well captured by the tube model [2] based on the reptation concept [3] in which the motion of a polymer chain in the isotropic network of entanglements with its neighboring chains can be understood as if the chain moves in a tube. While the basic tube model captures polymer physics in small deformations [4], even the most advanced versions [5] fail to describe entangled linear melts in strong flows [6]. The tube model assumes that the timescales for local and global relaxation phenomena, Rouse

Published by the American Physical Society under the terms of the Creative Commons Attribution 4.0 International license. Further distribution of this work must maintain attribution to the author(s) and the published article's title, journal citation, and DOI. relaxation $\tau_{R}$ and reptation $\tau_{d}$, scale with the degree of polymerization $N$ as $\tau_{R} \sim N^{2}$ and $\tau_{d} \sim N^{3.4}$. Until recently it was generally agreed that the separation of timescales was verified through small-angle neutron-scattering (SANS) experiments on polymer melts relaxing from small, sudden deformations such that the initial molecular deformation is affine with the macroscopic deformation. In this case, the molecules should initially retract in the deformed tube, i.e., diminish in all directions before they return to the isotropic equilibrium state [2]. The gyration tensor component perpendicular to elongation $R_{g}^{\perp}$ is particularly sensitive as it should increase nonmonotonically to its equilibrium value. Several SANS experiments tested the chain retraction hypothesis for small deformations up to a Hencky strain of $\varepsilon=\ln \left(l_{\text {final }} / l_{\text {initial }}\right) \approx$ 1.1 [7-9], where $l$ is the sample length. A nonmonotonic increase in $R_{g}^{\perp}$ was observed in [8,9] but not in Ref. [7], likely due to polydispersity. The inclusion of chain retraction in the model was also reported necessary to fit the SANS data in two studies of polymer deformation in a flow cell $[10,11]$.

However, Wang et al. [12] argued that spherical harmonics expansion of two-dimensional (2D) SANS patterns provides more sound evidence for chain retraction than a nonmonotonic increase in $R_{g}^{\perp}$. Chain retraction shows up as a dip shift in the first anisotropic expansion coefficient during the first $t \sim \tau_{R}$ of the stress relaxation. When applied to data for stress relaxation following a small sudden deformation 


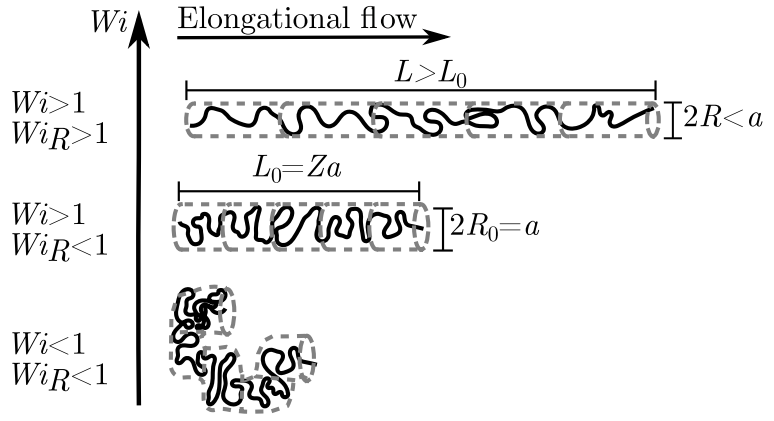

FIG. 1. Steady-state molecular conformation for elongation at increasing Weissenberg numbers $\mathrm{Wi}=\dot{\varepsilon} \tau_{d}$ and $\mathrm{Wi}_{R}=\dot{\varepsilon} \tau_{R}$, where $\dot{\varepsilon}$ is the deformation rate and $\tau_{d}$ and $\tau_{R}$ are the reptation and Rouse times, respectively. The $Z$ tube segments are marked by dashed lines.

of polystyrene with $Z \approx 34$ entanglement segments per chain stretched to $\varepsilon=0.6$, no dip shift occurred, which questions the chain retraction hypothesis.

Very recently O'Connor et al. [13] investigated stress relaxation in highly oriented melts of entangled polymers by molecular dynamics using a Kremer-Grest bead-spring model [14]. Results were shown for both relaxation of overall stress and several measures of tube length and diameter. Their simulations showed a striking separation between a rapid relaxation of molecular stretch on the timescale of the Rouse time and a subsequent slower configurational relaxation on the scale of the terminal relaxation time. Both the stress and molecular stretch exhibit the trend that the curve for a given rate merges onto the curves for larger rates during the Rouse relaxation process. The findings of O'Connor et al. are consistent with the experimental results of Nielsen et al. [15] on the nonlinear extensional rheology of linear polystyrene measured on a filament stretch rheometer that allowed for stretches to $\varepsilon=3$.

In this paper we show experimental evidence for the separation of $\tau_{R}$ and $\tau_{d}$ using spherical harmonics expansion of SANS data for polymer melt relaxation from a highly oriented melt. The highly oriented initial state is ensured by stretching the melt at a deformation rate that fulfills $\dot{\varepsilon}>$ $1 / \tau_{R}$. Due to the separation of $\tau_{R}$ and $\tau_{d}$ the molecules may orient or orient and stretch along the flow depending on the deformation rate $\dot{\varepsilon}$ relative to the characteristic relaxation times. This is also captured in the dimensionless Weissenberg numbers $\mathrm{Wi}=\dot{\varepsilon} \tau_{d}$ and $\mathrm{Wi}_{R}=\dot{\varepsilon} \tau_{R}$. When $\mathrm{Wi}<1$, reptation dominates and the polymer molecule is close to equilibrium. When $\mathrm{Wi}>1$ but $\mathrm{Wi}_{R}<1$, the steady flow situation is that of equilibrated polymer chains contained in $Z$ tube segments with length and diameter $a$ that are fully oriented along the stretching direction. Finally, when $\mathrm{Wi}>1$ and $\mathrm{Wi}_{R}>1$ the chains will exhibit stretching in the oriented tubes [16] as illustrated in Fig. 1. One should note that during the initial stress relaxation from this extreme stretched condition, one should not see the characteristic nonmonotonic relaxation of $R_{g}^{\perp}$, predicted for small deformations. According to the tube model, the relation $\tau_{d} \gg \tau_{R}$ would predict the stretched and oriented chain regaining the dimensions of the fully oriented chain during the first $t \sim \tau_{R}$ of the relaxation. We study
TABLE I. Weissenberg numbers $\mathrm{Wi}$ and $\mathrm{Wi}_{R}$. Experiments are carried out at $T=130{ }^{\circ} \mathrm{C}$

\begin{tabular}{lccc}
\hline \hline$\dot{\varepsilon}\left(\mathrm{s}^{-1}\right)$ & 0.003 & 0.03 & 0.1 \\
\hline $\mathrm{Wi}_{R}$ & 0.045 & 0.45 & 1.5 \\
$\mathrm{Wi}$ & 0.3 & 3 & 30 \\
\hline \hline
\end{tabular}

the relaxation of linear polystyrene of $M_{w}=80 \mathrm{~kg} / \mathrm{mol}$ and $Z \approx 5$ from the highly oriented state following an elongation at $\mathrm{Wi}_{R}=1.5>1$ to steady state. We show experimental evidence for separation of the Rouse and reptation times, or chain retraction, from this highly oriented state based on the dip shift in the first anisotropic expansion coefficient of the spherical harmonics expansion. The dip shift is consistent with the stretched molecules regaining the dimensions of the fully oriented tube within $t \sim \tau_{R}$ of the stress relaxation. In this way, we confirm and expand on the molecular dynamics simulations by O'Connor et al. and rheological measurements by Nielsen et al. with direct experimental observations of the average molecular conformation during the relaxation process.

\section{METHODS}

This paper extracts even further results from the combined rheological and structural study of a polystyrene sample, already published in [17]. Since the preparation of materials and the structural small-angle neutron-scattering experiments were presented in detail in [17], we will only repeat a few relevant details on the materials, their rheology, and the SANS experiments.

The material is a polystyrene blend of $90 \mathrm{wt}$. \% hydrogenous polystyrene with molecular weight $M_{w}=95 \mathrm{~kg} / \mathrm{mol}$ and polydispersity index (PDI) equal to 1.07 and $10 \mathrm{wt}$. $\%$ of $M_{w}=80 \mathrm{~kg} / \mathrm{mol}$ fully deuterated polystyrene with a PDI of 1.02. The extensional rheology of the hydrogenous component was previously studied and described by Hengeller et al. [18].

The extensional flow was obtained on a filament stretch rheometer (VADER-1000, Rheo Filament ApS) as described by Hengeller, Kirkensgaard and co-workers [17,18]. All samples were heated well above the glass transition temperature $T_{g} \approx 100^{\circ} \mathrm{C}$ to $T=130{ }^{\circ} \mathrm{C}$ and stretched at one of three constant Hencky strain rates $\dot{\varepsilon}$ to a final Hencky strain of $\varepsilon=3$ where steady flow was obtained [18]. This is a much larger deformation than any of the previous SANS experiments described above. The Weissenberg numbers for the stretches are listed in Table I and calculated from estimates of the Rouse and reptation times $\tau_{R} \approx 15 \mathrm{~s}$ and $\tau_{d} \approx 94 \mathrm{~s}$, respectively, for the deuterated molecules based on the results of Hengeller et al. [18].

The samples stretched at the two lowest Wi were cooled below the glass transition temperature to preserve molecular conformation for $e x$ situ scattering experiments as described by Hassager et al. [19] immediately after reaching $\varepsilon=3$. The samples stretched at the largest $\mathrm{Wi}$ were allowed to relax at the experimental temperature under constant Hencky strain for $t=0 \tau_{R}, 0.7 \tau_{R}, 1.3 \tau_{R}, 5.3 \tau_{R}$, and $21.3 \tau_{R}$ prior to the rapid 


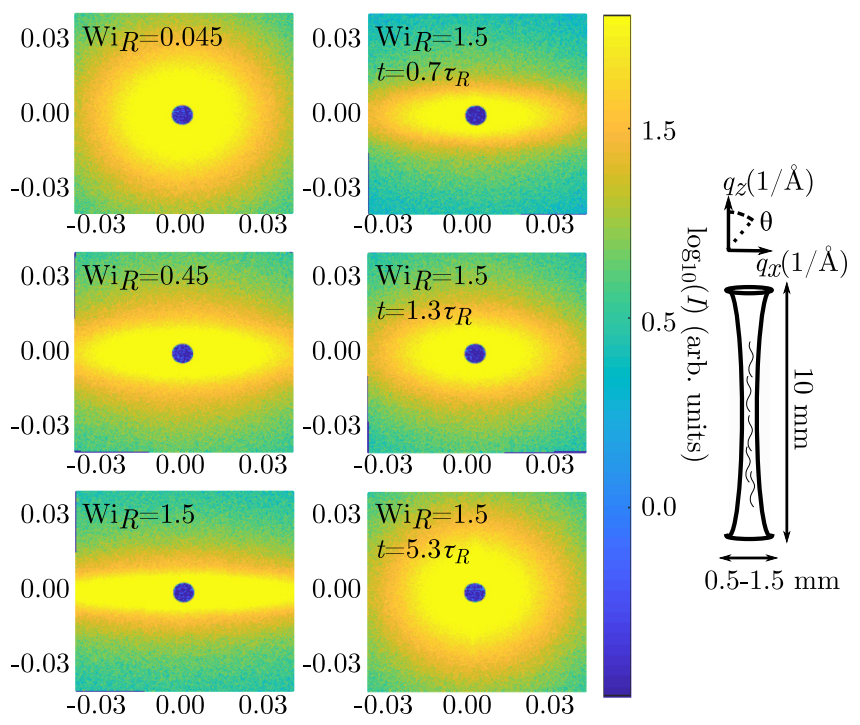

FIG. 2. Two-dimensional SANS data on uniaxially extended polystyrene and experimental geometry. Intensities are plotted on a logarithmic scale and the units of $q_{x}$ and $q_{z}$ are $1 \AA^{-1}$. The left column shows increasing $\mathrm{Wi}_{R}$ and the right column increasing $t$ following extension at $\mathrm{Wi}_{R}=1.5$. The fully relaxed pattern for $\mathrm{Wi}_{R}=1.5$ and $t=21.3 \tau_{R}$ is omitted. The stretching direction and polar angle $\theta$ are defined on the filament cartoon.

cooling. The cooling rate was $10 \mathrm{~K} / \mathrm{s}$ and the molecular conformation is essentially locked after approximately $3 \mathrm{~s}$, which is five times faster than $\tau_{R}$ at the experimental temperature. According to Fig. 1, we expect a molecular conformation close to equilibrium at the smallest $\mathrm{Wi}$, the tube segments to orient for intermediate $\mathrm{Wi}$, and the chain to stretch at the largest Wi [20].

Small-angle neutron-scattering data were collected on QUOKKA at the Australian Nuclear Science and Technology Organisation. The sample detector distances were 14, 5, and $2 \mathrm{~m}$, respectively and the neutron wavelength was $5 \AA$. The data from the $5-\mathrm{m}$ setting on the $\mathrm{Wi}_{R}=1.5$ samples were previously published in [17]. Each sample consists of several filaments and was measured with the filaments aligned vertically and perpendicular to the neutron beam, as sketched in Fig. 2. The data are reduced by subtracting blocked beam measurements.

The left column of Fig. 2 shows that the scattering patterns immediately after stretching change from isotropic to highly anisotropic with increasing $\mathrm{Wi}_{R}$. In the right column, the scattering patterns change from highly anisotropic to isotropic with increasing stress relaxation time all following a stretch at $\mathrm{Wi}_{R}=1.5$. The scattering patterns have a contribution from macroscopic cracks at the smallest scattering vector lengths $q$ (see Ref. [21] for further description).

The SANS data are analyzed using spherical harmonics expansion presented in Ref. [12]. For the experimental geometry (see Fig. 2), the measured scattering intensity $I\left(q_{x}, q_{y}=\right.$ $0, q_{z}$ ) can be decomposed in an orthogonal basis of spherical harmonics $Y_{l}^{m}(\theta)$ restricted to even $l$ and $m=0$ due to the symmetry of uniaxial extension,

$$
\begin{aligned}
I(\vec{q}) & =\sum_{l, \text { even }}^{\infty} I_{l}^{0}(q) Y_{l}^{0}(\theta), \\
Y_{l}^{0}(\theta) & =\sqrt{2 l+1} P_{l}^{0}(\cos \theta),
\end{aligned}
$$

where $I_{l}^{0}(q)$ are expansion coefficients, $P_{l}^{0}(x)$ are associated Legendre polynomials, $\theta$ is the polar angle in spherical coordinates that relates to the data as defined in Fig. 2, and $q=$ $|\vec{q}|=(4 \pi / \lambda) \sin (\vartheta)$ is the scattering vector length, where $\vartheta$ is half the scattering angle and $\lambda$ is the neutron wavelength. By expanding $I(\vec{q})$ in spherical harmonics, contributions to the scattering pattern are separated based on symmetry. The representation of $I(q)$ in terms of the expansion coefficients $I_{l}^{0}(q)$ is called the molecular fingerprint [12].

The separation of the isotropic and anisotropic expansion coefficients allows for a simple treatment of isotropic background, e.g., from incoherent scattering. An isotropic background only affects the isotropic expansion coefficient and can be estimated as the limit of $I_{0}^{0}(q)$ for large $q$ since $I_{l}^{0}(q) \rightarrow 0$ for $q \rightarrow \infty$ for all $l$ for the coherent part of $I$. We scale the 2D patterns to compensate for varying material amount across samples by estimating the incoherent scattering in this way.

\section{DISCUSSION AND CONCLUSION}

To study the molecular deformation, we focus on the expansion coefficient of the lowest-order anisotropic term $I_{2}^{0}(q)$ with the symmetry of uniaxial extension. As we see in Fig. $3, I_{2}^{0}(q)$ is negative for most samples and has a minimum. Figure 3(a) shows that the minimum in $I_{2}^{0}(q)$ deepens and shifts towards smaller $q$ for increasing Wi and Fig. 3(b) shows that the minimum flattens out and shifts towards larger $q$ with increasing stress relaxation time. For the $\mathrm{Wi}=0.045$ and the $\mathrm{Wi}=1.5$ and $t=21.3 \tau_{R}$ samples, the $I_{2}^{0}(q)$ curves are essentially flat, consistent with an almost isotropic equilibrium conformation. In the following, we take the position of the minimum in $I_{2}^{0}(q)$ to reflect molecular conformation. As a consequence, a dip shift indicates a change in molecular conformation.

To quantify the minimum shift, we compare to a simple model of the fully oriented tube, namely, a cylinder with uniform density. The form factor for an oriented cylinder is

$$
P(\vec{q})=\left|\frac{\sin \left(q_{\|} L / 2\right)}{q_{\|} L / 2} 2 \frac{J_{1}\left(q_{\perp} R\right)}{q_{\perp} R}\right|^{2},
$$

where $L$ is the cylinder length, $R$ is the cylinder radius, $q_{\|}$ $\left(q_{\perp}\right)$ is the component of the scattering vector $\vec{q}$ parallel (perpendicular) to the cylinder axis, and $J_{1}(x)$ is a Bessel function of the first kind. The form factor can be found from, e.g., Ref. [22] by omitting an orientational average. For the fully oriented tube, we choose the radius to be half the tube diameter $R_{0}=a / 2$ and the cylinder length $L_{0}$ to be given by the number of entanglement segments $Z$ as $L_{0}=Z a$. We use molecular parameters for polystyrene [23] to set $R_{0}=a / 2=42.5 \AA$ and find $Z$ as the ratio of the 

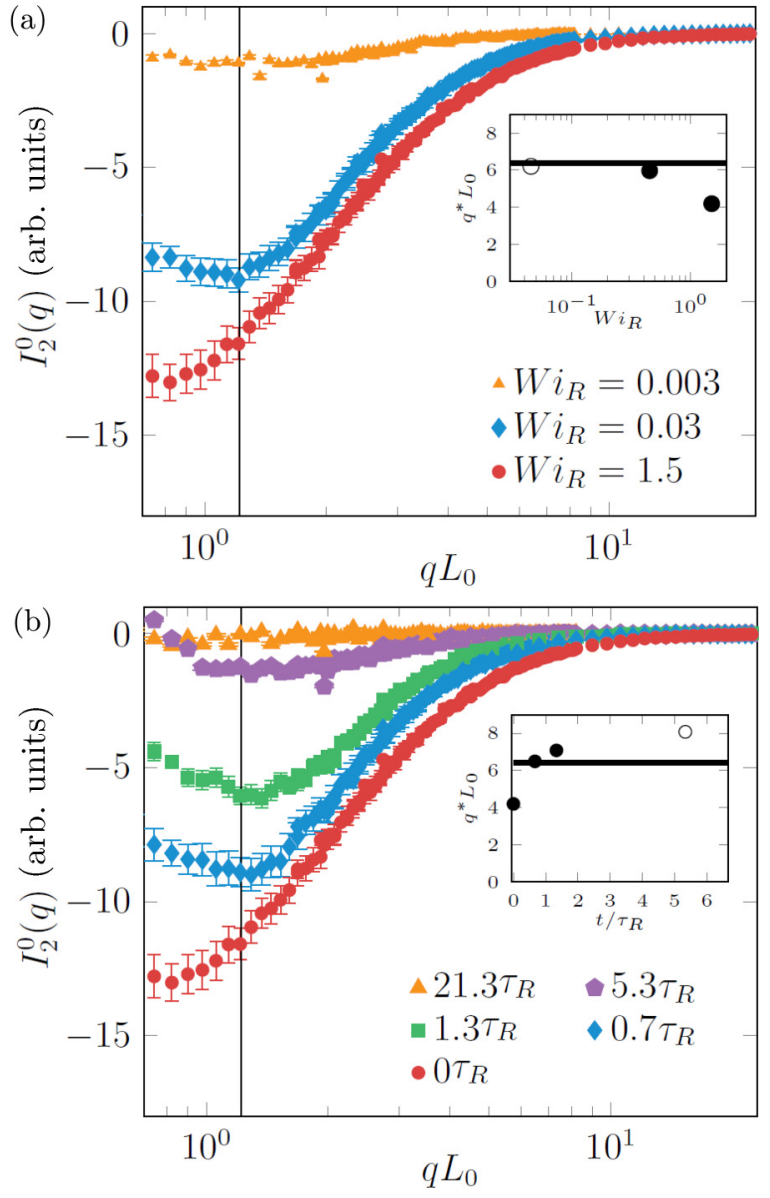

FIG. 3. Expansion coefficient $I_{2}^{0}(q)$ as a function of scattering vector made dimensionless with the length of the fully oriented tube $q L_{0}$ for (a) varying Rouse-Weissenberg number $\mathrm{Wi}_{R}$ and (b) varying duration of stress relaxation $t$ following a deformation with $\mathrm{Wi}_{R}=$ 1.5. Vertical lines show the location of the minimum for the fully oriented chain from the cylinder model. The insets show $q^{*}$ that minimizes $I_{2}^{0}(q)$ (a) as a function of $\mathrm{Wi}_{R}$ and (b) as a function of $t$ relative to the Rouse time $\tau_{R}$. Open symbols indicate an uncertain value due to shallowness or crack scattering. The fully drawn horizontal lines show the minimum position for the fully oriented chain. All samples are stretched to a final Hencky strain of $\varepsilon=3$.

molecular weight and the entanglement molecular weight $Z=M_{w} / M_{e}=80(\mathrm{~kg} / \mathrm{mol}) / 16.6(\mathrm{~kg} / \mathrm{mol}) \approx 5$. This yields $L_{0} \approx 410 \AA$ for the fully oriented chain. We expand $P(\vec{q})$ in spherical harmonics and determine the minimum of $P_{2}^{0}(q)$. It can be shown by direct calculations that the position of the minimum is far more sensitive to relative variations in the cylinder length than radius and we take the minimum position of the experimental data to reflect the molecular length.

To study chain stretch and its relaxation, we plot $q^{*}$ that minimizes $I_{2}^{0}(q)$ as a function of the Rouse-Weissenberg number $\mathrm{Wi}_{R}$ and stress relaxation duration $t$, respectively, in the insets of Fig. 3. We compare $q^{*}\left(\mathrm{Wi}_{R}, t\right)$ to the minimum of $P_{2}^{0}(q)$ for the fully oriented tube. For the intermediate $\mathrm{Wi}_{R}, q^{*}$ is consistent with the minimum of $P_{2}^{0}(q)$, although it suggests a slightly larger elongation. For the largest $\mathrm{Wi}_{R}$, which fulfills $\mathrm{Wi}_{R}>1$, the minimum shifts to an even smaller value of $q$. This shows that not only is the tube fully oriented, but

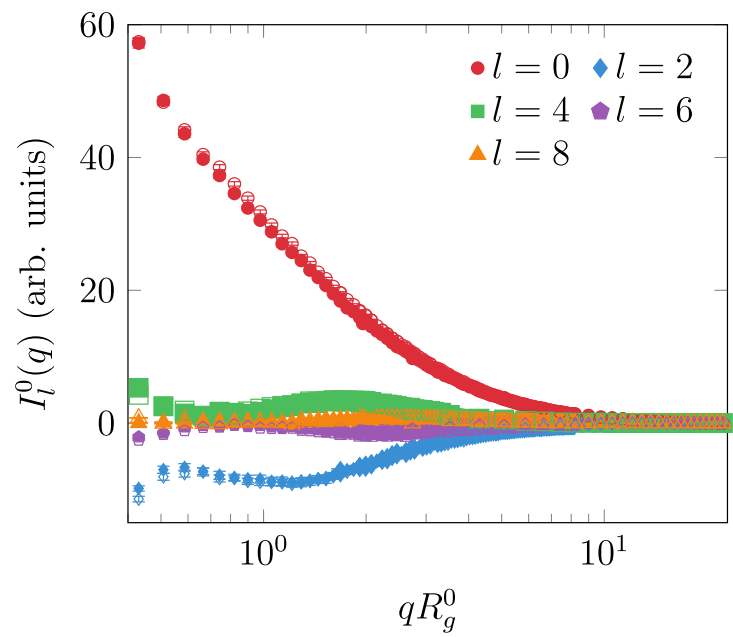

FIG. 4. Comparison of the overlapping fingerprints for molecular conformations with a cylinder length slightly larger than $L_{0}, \mathrm{Wi}_{R}=$ 0.15 and $t=0.7 \tau_{R}\left(\mathrm{Wi}_{R}=0.45\right.$ and $\left.\mathrm{Wi}=3\right)$, in closed (open) symbols.

the chain is also markedly stretched beyond the equilibrium chain length by the flow. The value of $q^{*}$ is consistent with a cylinder length of $L \approx 440 \AA$, i.e., the chain is stretched about $7 \%$ relative to the fully oriented tube segments without chain stretch. If we now turn to the relaxation in Fig. 3(b), we see that the minimum shifts to a $q$ value close to but slightly above the one predicted by the cylinder model after $t \approx \tau_{R}$ corresponding to a molecular conformation slightly less elongated than the fully oriented tubes as modeled by the cylinder. That chain stretch occurs for $\mathrm{Wi}_{R}>1$ and that it relaxes in $t \sim \tau_{R}$ shows that $\tau_{R}$ and $\tau_{d}$ are separated, or that chain retraction occurs.

The molecular elongation is very similar for both samples that are expected to correspond to fully oriented cylinders, for $\mathrm{Wi}_{R}=0.45$ and for $\mathrm{Wi}_{R}=1.5$ and $t=0.7 \tau_{R}$, and we would expect overall similar conformations, provided there is no remaining stretching. Differences would appear if we compare the full fingerprint and not just the minimum position of $I_{2}^{0}(q)$. The fingerprints of the $\mathrm{Wi}_{R}=0.45$ and of the $\mathrm{Wi}_{R}=1.5$ and $t=0.7 \tau_{R}$ in Fig. 4 overlap, showing indeed that not only is the molecular elongation the same but so is their average molecular conformation. This result is in accordance with the average molecular conformations as a function of $\mathrm{Wi}_{R}$ and stress relaxation time $t$ extracted from the molecular dynamics simulations of O'Connor et al. [13].

Our results show that chain retraction can be observed with spherical harmonics expansion by a shift in the first anisotropic expansion coefficient even for short polymer chains provided the initial state is highly oriented due to $\mathrm{Wi}_{R}>1$ and a sufficiently large deformation. For the experiments by Wang et al. [12] and the follow-up simulations with comparable parameters in [24], the final Hencky strain was rather small, $\varepsilon=0.6$, and the molecules were rather short compared to the systems described in the beginning where chain retraction was observed. That could explain the absence of signs of chain retraction. This explanation was also proposed by O'Connor et al. and Hsu and Kremer [25]. The observation of chain retraction shows that the 
molecules keep the memory of the equilibrium network of entanglements or recover it during the first $t \sim \tau_{R}$ of stress relaxation, which was also seen for three armed stars in Ref. [21].

To conclude, spherical harmonics expansion of small-angle neutron-scattering data demonstrates chain stretch and relaxation in highly oriented polymer melts. Chain stretch was observed above fully oriented tube segments for $\mathrm{Wi}_{R} \geqslant 1$ and this chain stretch was seen to relax to fully oriented tube segments in time $t \sim \tau_{R}$. This shows the separation of Rouse and reptation times as described by the tube model. The results presented in this paper directly show the evolution of molecular conformation with stress relaxation as proposed in previous rheology and molecular dynamics works. The spherical harmonics expansion allows for detailed examination of anisotropic components and it will be interesting to see what insights into polymer dynamics can be gained by analyzing, or reanalyzing, other scattering patterns with spherical harmonics expansion.

\section{ACKNOWLEDGMENTS}

The authors would like to thank Daniel Read for fruitful discussions, ANSTO for beamtime, DanScatt, Danish Council for Independent Research, Aage og Johanne Louis-Hansens Fond and Villum Fonden for funding (Grant No. 17363).
[1] S. Chandran, J. Baschnagel, D. Cangialosi, K. Fukao, E. Glynos, L. M. C. Janssen, M. Müller, M. Muthukumar, U. Steiner, J. Xu, S. Napolitano, and G. Reiter, Macromolecules 52, 7146 (2019).

[2] M. Doi and S. F. Edwards, J. Chem. Soc. Faraday Trans. 2 74, 1802 (1978).

[3] P.-G. de Gennes, J. Chem. Phys. 55, 572 (1971).

[4] T. C. B. McLeish, Adv. Phys. 51, 1379 (2002).

[5] R. S. Graham, A. E. Likhtman, T. C. B. McLeish, and S. T. Milner, J. Rheol. 47, 1171 (2003).

[6] D. C. Venerus, R. M. Mick, and T. Kashyap, J. Rheol. 63, 157 (2019).

[7] F. Boué, M. Nierlich, G. Jannink, and R. Ball, J. Phys. (Paris) 43, 137 (1982).

[8] K. Mortensen, O. Kramer, W. Batsberg, and L. J. Fetters, MRS Proc. 79, 259 (1986).

[9] A. Blanchard, R. S. Graham, M. Heinrich, W. PyckhoutHintzen, D. Richter, A. E. Likhtman, T. C. B. McLeish, D. J. Read, E. Straube, and J. Kohlbrecher, Phys. Rev. Lett. 95, 166001 (2005).

[10] J. Bent, L. R. Hutchings, R. W. Richards, T. Gough, R. Spares, P. D. Coates, I. Grillo, O. G. Harlen, D. J. Read, R. S. Graham, A. E. Likhtman, D. J. Groves, T. M. Nicholson, and T. C. B. McLeish, Science 301, 1691 (2003).

[11] R. S. Graham, J. Bent, L. R. Hutchings, R. W. Richards, D. J. Groves, J. Embery, T. M. Nicholson, T. C. B. McLeish, A. E. Likhtman, O. G. Harlen, D. J. Read, T. Gough, R. Spares, P. D. Coates, and I. Grillo, Macromolecules 39, 2700 (2006).
[12] Z. Wang, C. N. Lam, W.-R. Chen, W. Wang, J. Liu, Y. Liu, L. Porcar, C. B. Stanley, Z. Zhao, K. Hong, and Y. Wang, Phys. Rev. X 7, 031003 (2017).

[13] T. C. O'Connor, A. Hopkins, and M. O. Robbins, Macromolecules 52, 8540 (2019).

[14] K. Kremer and G. S. Grest, J. Chem. Phys. 92, 5057 (1990).

[15] J. K. Nielsen, H. K. Rasmussen, and O. Hassager, J. Rheol. 52, 885 (2008).

[16] G. Ianniruberto and G. Marrucci, Macromolecules 46, 267 (2013).

[17] J. J. K. Kirkensgaard, L. Hengeller, A. Dorokhin, Q. Huang, C. J. Garvey, K. Almdal, O. Hassager, and K. Mortensen, Phys. Rev. E 94, 020502(R) (2016).

[18] L. Hengeller, Q. Huang, A. Dorokhin, N. Alvarez, K. Almdal, and O. Hassager, Rheol. Acta 55, 303 (2016).

[19] O. Hassager, K. Mortensen, A. Bach, K. Almdal, H Rasmussen, and W. Pyckhout-Hintzen, Rheol. Acta 51, 385 (2012).

[20] G. Ianniruberto and G. Marrucci, J. Rheol. 45, 1305 (2001).

[21] K. Mortensen, A. L. Borger, J. J. K. Kirkensgaard, C. J. Garvey, K. Almdal, A. Dorokhin, Q. Huang, and O. Hassager, Phys. Rev. Lett. 120, 207801 (2018).

[22] J. Pedersen, Adv. Colloid Interface Sci. 70, 171 (1997).

[23] L. J. Fetters, D. J. Lohse, and R. H. Colby, in Physical Properties of Polymers Handbook, 2nd ed., edited by J. E. Mark (Springer, New York, 2007), Chap. 25, pp. 447-454.

[24] W.-S. Xu, J.-M. Y. Carrillo, C. N. Lam, B. G. Sumpter, and Y. Wang, ACS Macro Lett. 7, 190 (2018).

[25] H.-P. Hsu and K. Kremer, Phys. Rev. Lett. 121, 167801 (2018). 